\title{
Antibody loaded collapsible hyaluronic acid hydrogels \\ for intraocular delivery
}

Raphael Egbu', Steve Brocchini ${ }^{1}, 2$, Peng T. Khaw ${ }^{2}$ and Sahar Awwad ${ }^{1,2 *}$

${ }^{1}$ UCL School of Pharmacy, London, WC1N 1AX, UK

${ }^{2}$ National Institute for Health Research (NIHR) Biomedical Research Centre at Moorfields Eye Hospital NHS Foundation Trust and UCL Institute of Ophthalmology, London, EC1 V9EL, UK

Keywords: hyaluronic acid, protein, ocular drug delivery, vitreous, controlled release, thermoresponsive

Corresponding author: s.awwad@ucl.ac.uk 


\begin{abstract}
Injectable gels have the potential to encapsulate drugs for sustained release of protein therapeutics for use in the eye. Hyaluronic acid $(\mathrm{HA})$ is a biodegradable clinically used material and poly $\mathrm{N}$-isopropylacrylamide (pNIPAAM) is a stimuli responsive polymer that can display a lower critical solution temperature (LCST) at physiological conditions. Two gel systems incorporating HA were prepared in the presence of the antibody infliximab (INF): i) $1 \%$ and $5 \%$ tyramine-substituted HA (HA-Tyr) was enzymatically crosslinked in the presence of INF to form HA-Tyr-INF and ii) NIPAAM was chemically crosslinked in the presence of $\mathrm{HA}$ and INF with 1 and $3 \%$ poly(ethylene glycol) diacrylate (PEGDA) to form PEGDA-pNIPAAM-HA-INF. The PEGDA-pNIPAAM-HAINF hydrogels displayed LCSTs at temperatures ranging from $31.4 \pm 0.2$ to $35.7 \pm$ $0.3^{\circ} \mathrm{C}$. Although all the gels prepared were injectable, INF-loaded gels with lower crosslinking density (1\% PEGDA-pNIPAAM-HA and 1\% HA-Tyr) showed lower elastic $\left(G^{\prime}\right)$ and viscous $\left(G^{\prime \prime}\right)$ moduli compared to higher crosslinked gels (3\% PEGDApNIPAAM-HA-INF and 5\% HA-Tyr-INF) resulting in differences in swelling ratio (SR). Moduli may be correlated with overall stiffness of the gel. All hydrogels demonstrated sustained release of INF in a two-compartment in vitro outflow model of the human eye called the PK-Eye. The 1\% PEGDA-pNIPAAM-HA-INF hydrogel displayed the slowest release $(24.9 \pm 0.4 \%$ INF release by day 9$)$ in phosphate buffered saline (PBS, $\mathrm{pH} 7.4$ ), which is a better release profile than the free drug alone (tested under the same conditions). These results suggest that PEGDA-pNIPAAM-HA has potential for the continued development of formulations to prolong the intraocular release of proteins.
\end{abstract}




\section{Introduction}

Many new therapies including therapeutic proteins and cellular therapies are being developed to treat blinding diseases for an ageing population. As molecular targets for disease continue to be identified, therapeutic proteins are being intensively developed because they tend to be fast acting, selective and potent. Currently, antibodies to treat age related macular degeneration (AMD) are administered every 4-8 weeks by intravitreal (IVT) injections. New dosage forms and drug delivery systems (DDSs) that extend the presence of an IVT administered drug are highly sought to (i) to reduce the frequency of administration, cumulative doses and undesirable side effects, (ii) to ensure a therapeutic dose of a drug is present throughout treatment and (iii) to improve patient compliance. ${ }^{1,2}$

In situ forming hydrogels have been shown to be a promising method to extend the duration of protein. ${ }^{3}$ Hydrogels can mimic the structure of the extracellular matrix (ECM) and have been shown to be biocompatible. ${ }^{4}$ These three dimensional (3D) polymer networks are capable of absorbing a large amount of water and have been examined in a number of tissue regeneration applications. ${ }^{4}$ Hydrogels can be formed by physical (e.g. hydrophobic, charge interaction) or chemical crosslinking (e.g. covalent) of polymers. Interpenetrating polymer networks (IPN) consists of a mixture of two or more polymers and can include polymer networks where one component is at least partially interlaced on a molecular segmental scale but not covalently bonded to each other. ${ }^{5}$ Semi-IPNs avoid the restrictions of excessive interpenetrating network and can maintain rapid response to external stimuli e.g. temperature change to change polymer conformation while extending release profile. ${ }^{6}$

Hyaluronic acid (HA) is a pseudo-elastic material with shear thinning properties that is capable of gelation. HA stabilises and organises the ECM, regulates cell adhesion and motility and mediates cell proliferation and differentiation. ${ }^{7}$ The commercially available form, sodium hyaluronate, has been widely used in pharmaceuticals for biomedical applications such as scaffolds for wound healing and tissue engineering due to its biocompatible and biodegradable properties. ${ }^{8,9} \mathrm{HA}$ is also used in ophthalmic surgery and as a component in implants. ${ }^{8}$ Unmodified HA is used as an adjuvant for ophthalmic drug delivery and enhances the absorption of drugs and proteins via the mucosal tissue. ${ }^{10} \mathrm{HA}$ is also used in the fields of viscosurgery and viscosupplementation. ${ }^{11} \mathrm{HA}$ is clinically approved for use in eye surgeries including cataract removal and corneal transplantation for the repair of a detached retina and other eye injuries. HA has been used to prepare IPNs ${ }^{12}$ and collapsible hydrogels ${ }^{8,13-}$ ${ }^{16}$ by either chemically modifying ${ }^{13,17,18}$ or chemically crosslinking. ${ }^{14,16,19-22}$ 
HA can be functionalised with tyramine (Tyr) and is commercially available as Corge ${ }^{\circledR}$ BioHydrogel (HA-Tyr) with Tyr substitution from 1-5\% on a molar basis. Tyr moieties can then be covalently linked using horseradish peroxidase (HRP) resulting in a crosslinked HA hydrogel. ${ }^{17,18,21-25}$ Crosslinking $\mathrm{HA}$ in the presence of the therapeutic antibody provides a means to ensure the antibody is mixed in the hydrogel. Incubation of an antibody with a hydrogel will not result in sufficient entanglement or encapsulation that could be required to prolong the release of the antibody from the gel.

The use of a collapsible thermoresponsive hydrogel provides the potential for further prolongation of antibody release from an injected hydrogel formulation. Poly $(\mathrm{N}-$ isopropylacrylamide) (pNIPAAM) is a thermoresponsive polymer ${ }^{26-33}$ that is biocompatible, but it is not biodegradable. ${ }^{34}$ Incorporation of a HA biodegradable component ${ }^{35,36}$ as an IPN in the pNIPAAM network is one strategy to allow the clearance of pNIPAAM. ${ }^{21,34}$ It is possible that when the biodegradable component HA is incorporated within pNIPAAM, the degradation of $\mathrm{HA}$ will reduce the overall polymer network allowing pNIPAAM oligomers to be soluble and excreted.

Here we report the preparation of two types of HA hydrogels that have been loaded with infliximab (INF) as a model protein. INF binds to tissue necrosis factor alpha (TNF- $\alpha$ ). Antibodies that target TNF- $\alpha$ have the potential to be used to treat ocular inflammation. ${ }^{37-39}$ The hydrogels were (i) a semi-IPN called PEGDA-pNIPAAMHA where NIPAAM was chemically crosslinked in presence of $H A$ and INF with 1 and $3 \%$ poly(ethylene glycol) diacrylate (PEGDA) and ii) an enzymatically crosslinked HATyr-INF that consisted of $1 \%$ and $5 \%$ tyramine-substituted HA (HA-Tyr) with INF. Both types of hydrogel were prepared in the presence of INF to ensure the antibody was entrapped within the hydrogel network. All hydrogels were characterised for their rheology, thermoresponsiveness and release kinetics using a two-compartment in vitro outflow model of the human eye (the PK-Eye)..$^{40}$

\section{Materials and Method}

\section{Materials}

Infliximab (Remicade ${ }^{\circledR} 100 \mathrm{mg} / 10 \mathrm{~mL}$, Genentech, South San Francisco, California) was obtained from the pooled leftovers from Great Ormond Street Hospital (London, UK). $N$-isopropylacrylamide (NIPAAM), azobisisobutyronitrile (AIBN), ammonium persulfate (APS), sodium azide, poly(ethylene glycol) diacrylate (PEGDA, Mn: 575) and tetramethylethylene-diamine (TEMED) were purchased from Sigma-Aldrich (Gillingham, Dorset, UK). Tyramine-substituted (1 and 5\%) hyaluronic acid (Corgel Biohydroge ${ }^{\circledR}$ kit) was purchased from Lifecore Biomedical, LLC (Chaska, Minnesota, 
USA). Eye drop grade hyaluronic acid (HA, $50 \mathrm{kDa}$ ) was purchased from Zhenjiang Group, China. Phosphate Buffer Saline (PBS) tablets was purchased from Fisher Scientific (Loughborough, Leicestershire, UK). Pierce ${ }^{\mathrm{TM}}$ micro bicinchoninic acid (BCA) protein assay kit was purchased from Thermo Fisher Scientific (Hertfordshire, UK). Visking dialysis membrane tubing (MWCO of 12-14 kDa) was purchased from Medicell International Ltd. (London, UK).

\section{Instrumentation}

A 16-channel Ismatec peristaltic pump (Michael Smith Engineers Ltd., Woking, Surrey, UK) was used to maintain fluid flow through the PK-Eye. UV measurements were performed using a Hitachi U-2800A spectrometer using Quartz cuvettes (Starna Scientific Ltd) with a wavelength range of 200 to $800 \mathrm{~nm}$ and a Wallac Victor2 1420 plate reader. Scanning electron microscopy (SEM) was achieved using a QuantaTM 200F instrument (FEI Quanta200 FEGESEM, Eindhoven, The Netherlands). Fouriertransform infrared spectroscopy (FTIR) was recorded with Perkin-Elmer Spectrum 100 FTIR spectrometer and analysed using Perkin-Elmer Spectrum Express software. Differential scanning calorimetry (DSC) experiments were performed on DSC Q2000 (TA instruments, Waters, LLC) with TA Instruments Universal Analysis 2000 software. A VIRTIS-Advantage freeze-dryer was used for freeze-drying. Bohlin CVO rotational rheometer (Malvern Instruments Ltd, Malvern, UK) was used to study rheological properties of the hydrogels. An Innova ${ }^{\circledR} 40$ incubator (New Brunswick Scientific, Edison, USA) was used for incubation of the samples.

\section{Methods}

\section{Hydrogel preparation}

\section{HA-Tyr gel}

HA-Tyr powder (4.8 kDa, confirmed by matrix-assisted laser desorption/ionisation (MALDI-TOF), 1 or $5 \%$ Tyr substitution, $10.0 \mathrm{mg}, 2.1 \times 10^{-6}$ moles) was completely dissolved in deionised water $(0.5 \mathrm{~mL})$. HRP in PBS $(0.5 \mathrm{~mL}, 10.0 \mathrm{UI} / \mathrm{mL})$ was then added dropwise over a 10 second period and the reaction solution was gently mixed. Hydrogen peroxide $\left(\mathrm{H}_{2} \mathrm{O}_{2}\right)$ in PBS ( $80 \mu \mathrm{L}, 2.4 \times 10^{-5}$ moles) was then added and the reaction mixture was incubated at $4^{\circ} \mathrm{C}$ for 24 hours to ensure complete cross-linking and polymerisation. INF solution $(0.5 \mathrm{~mL}$ from $10.0 \mathrm{mg} / \mathrm{mL}$ to give a final protein concentration of $5.0 \mathrm{mg} / \mathrm{mL}$ ) was substituted for deionised water to dissolve the HATyr for the preparation of INF loaded hydrogels.

The reaction mixture was either stored in the fridge $\left(4^{\circ} \mathrm{C}\right)$ or freeze-dried for characterisation (such as fourier transform infrared spectroscopy (FTIR), scanning 
electron microscopy (SEM) and swelling studies) in tarred $2.0 \mathrm{~mL}$ glass vials and the product was isolated as a dry, solid white cake. The hydrogels were transferred to glass vials with a maximum volume of $2.0 \mathrm{~mL}$ for freeze-drying. The samples were frozen in dry ice and then transferred to the freeze-drier at $-40^{\circ} \mathrm{C}$ with a condenser temperature of $-60^{\circ} \mathrm{C}$. The vacuum pressure was maintained less than $200 \mathrm{mBar}$. The primary freeze-drying step was at $-20^{\circ} \mathrm{C}$ for 48 hours. The temperature was increased to $20^{\circ} \mathrm{C}$ for 2 hours at the final stage before opening the freeze-drier.

\section{PEGDA-pNIPAAM-HA gel}

NIPAAM (40.0 mg, $3.5 \times 10^{-4}$ moles) and HA (50 kDa, $\left.10.0 \mathrm{mg}, 2.0 \times 10^{-7} \mathrm{moles}\right)$ were dissolved in deionised water $(1.0 \mathrm{~mL})$ in a $7.0 \mathrm{~mL}$ vial. PEGDA (Mn 575) was added $\left(2.5 \mu \mathrm{L}, 1.0 \times 10^{-5}\right.$ moles and $5 \mu \mathrm{L}, 5.0 \times 10^{-6}$ moles corresponding to 1 and $3 \%$ PEGDA respectively) and gently swirled to ensure mixing. APS (4.0 mg, $1.8 \times 10^{-5}$ moles, 0.05 equiv) and TEMED ( $\delta: 0.775 \mathrm{~g} / \mathrm{mL}, 20^{\circ} \mathrm{C}, 20 \mu \mathrm{L}, 1.0 \times 10^{-4}$ moles, 0.29 equiv) were added to the reaction mixture and the vial was capped then the solution was gently vortexed for 30 seconds. The mixture was incubated in the fridge at $4^{\circ} \mathrm{C}$ for 24 hours to allow NIPAAM polymerisation and crosslinking to occur. For the INF loaded hydrogels, $0.5 \mathrm{~mL}$ of the deionised water used to dissolve NIPAAM and HA was substituted with INF solution $(0.5 \mathrm{~mL}, 10 \mathrm{mg} / \mathrm{mL})$. The reaction mixture was either stored in the fridge $\left(4^{\circ} \mathrm{C}\right)$ or freeze-dried as previously detailed.

\section{Hydrogel characterisation}

\section{Fourier transform infrared spectroscopy (FTIR)}

Freeze-dried samples were measured with attenuated total reflection (ATR). The sample $(0.25 \mathrm{~mL}$ reaction mixture) was pressed against a high refractive index prism. Absorption was measured for wavenumbers between 650 to $4000 \mathrm{~cm}^{-1}$ with 20 scans at a resolution of $2.0 \mathrm{~cm}^{-1}$.

\section{Scanning electron microscopy (SEM)}

Freeze-dried samples $(1.0 \mathrm{~mL}$ reaction mixture) were cut and adhered onto aluminium SEM stubs using carbon-coated double-sided tape prior to SEM analysis. The samples were then sputter coated with gold prior to imaging to make them electrically conductive.

\section{Differential scanning calorimetry (DSC)}

The lower critical solution temperature (LCST) was measured using differential scanning calorimetery (DSC) at $2.0^{\circ} \mathrm{C} / \mathrm{min}$ from 20 to $50^{\circ} \mathrm{C}$. Freeze-dried hydrogels ( $1.0 \mathrm{~mL}$ reaction mixture) were transferred to a $7.0 \mathrm{~mL}$ glass vial and swollen in water 
for 24 hours. The water was emptied and the hydrogels were transferred to a weighing container. The hydrogel was carefully and gently dabbed dry with tissue and then placed in aluminium hermetic pans of known weight. Calibration with Indium (Tm = 156.6, $\Delta \mathrm{Hf}=28.71 \mathrm{~J} / \mathrm{g}$ ) was performed according to the manufacturer instructions. Nitrogen was used as purge gas with a flow rate of $40.0 \mathrm{~mL} / \mathrm{min}$ for all the experiments. The onset temperature of the DSC endothermic peak was considered as the LCST.

\section{Rheology}

The rheological behavior of the hydrated hydrogels $(1.0 \mathrm{~mL}$ reaction mixture) was investigated under oscillatory shear. A $20 \mathrm{~mm}$ diameter plate was used for the analysis under auto-stress. Initially amplitude sweep feature determined the linear viscoelastic region (LVER) before frequency sweeps at $0.1-10 \mathrm{~Hz}$ were performed at an isothermal temperature of $25^{\circ} \mathrm{C}$. Furthermore, the influence of temperature on the rheology of the gels was determined by applying oscillatory shear at a fixed frequency $(1 \mathrm{~Hz})$ across a temperature range $\left(20-50^{\circ} \mathrm{C}\right)$. The mechanical properties were described in terms of elastic modulus ( $G^{\prime}$, Equation 1a) or viscous modulus ( $G^{\prime \prime}$, Equation $1 b)$. Complex modulus $\left(G^{*}\right)$ measures the gel's overall rigidity or resistance to deformation (Equation 1c).

\section{Equation 1}

$$
\begin{gathered}
G^{\prime}=\frac{\text { stress }}{\text { strain }} \cos \theta \\
G^{\prime \prime}=\frac{\text { stress }}{\text { strain }} \sin \theta \\
G *=G^{\prime}+i G^{\prime \prime}
\end{gathered}
$$

\section{Swelling ratio (SR)}

Freeze-dried hydrogel samples $(1.0 \mathrm{~mL}$ reaction mixture) were weighed and incubated in deionised water $(2.0 \mathrm{~mL})$ at 25 or $37^{\circ} \mathrm{C}$ for 48 hours. The samples were then lightly wiped and re-weighed. SR was calculated according to the Equation 2, where Ms is the weight of swollen gel and $M d$ is the weight of dry hydrogel.

$$
S R=\frac{M s-M d}{M d}
$$

\section{Equation 2}

\section{In vitro release}

The design and validation of the in vitro PK-Eye model has been previously reported..$^{40}$ The model comprises of an anterior $(0.2 \mathrm{~mL})$ and posterior $(4.2 \mathrm{~mL})$ cavity integrated within it. A washer with a Visking membrane with a MWCO 12-14 kDa separates the two cavities. The inlet port was connected to a peristaltic pump (previously calibrated) 
with a flow of $2.0 \mu \mathrm{L} / \mathrm{min}$. One outlet port is present in the anterior cavity for continuous sampling. An injection port is present in both cavities (diameter: 2.0-3.0 mm) to allow formulation administration into the model. Prior to each experiment, the PK-Eye was disassembled and the washer with a fresh Visking membrane was secured in the model separating the anterior and posterior cavities. Each model was assembled and both cavities were filled with PBS $(\mathrm{pH}$ 7.4). The models were immersed in a preheated oil bath adjusted to $37^{\circ} \mathrm{C}$ for 2 hours to equilibrate prior to the release studies. Temperature was maintained using a probe connected to the hotplate heater. INFloaded hydrogel $(1.0 \mathrm{mg}, 200 \mu \mathrm{L})$ was injected via a $21 \mathrm{G}$ needle via the injection port of the PK-Eye. Samples were collected from the outflow port at regular intervals and stored at $4^{\circ} \mathrm{C}$ prior to protein quantification.

\section{Protein quantification}

Protein quantification was conducted with a micro BCA protein assay kit. All released samples from the PK-Eye and standards $(100 \mu \mathrm{L})$ were analysed in a Nunc 96-well plate using BCA working reagent $(100 \mu \mathrm{L})$. A stock solution of INF $(200 \mu \mathrm{g} / \mathrm{mL})$ was prepared and serial dilutions with PBS $(\mathrm{pH} 7.4)$ were made $(0-200 \mu \mathrm{g} / \mathrm{mL})$. The plates were then incubated at $37^{\circ} \mathrm{C}$ for 2 hours before UV quantification. A calibration curve was plotted $\left(R^{2}: 0.9998\right)$ at $562 \mathrm{~nm}$ with a 96 well plate reader and unknown concentrations from the PK-Eye were determined by the equation generated. Average absorbance of blank $(0 \mu \mathrm{g} / \mathrm{mL})$ was subtracted from each reading.

\section{Data analysis}

All results are presented as the mean and standard deviation ( \pm STD), and data were plotted using OriginPro 9.1 (software, Origin lab cooperation, USA). Half-life $\left(t_{1 / 2}\right)$ values were calculated according to the best fitting model in OriginPro. Statistical significance was determined using one-way ANOVA or Mann Whitney $U t$-test. Probability values less than $0.05(p<0.05)$ were considered as indicative of statistically significant differences.

\section{Results and discussion}

\section{Preparation of hydrogels}

Two types of HA in situ hydrogels were prepared. The first, HA-Tyr hydrogel had been previously investigated as a biocompatible drug delivery material. ${ }^{41}$ The effect of crosslinking density on the behaviour of the HA-Tyr hydrogels was investigated based on the degree of Tyr substitution of HA (1 and 5\%). The second, a pNIPAAM-based gel was chosen because it possesses thermoresponsive/collapsing property and was 
expected to sustain the release of the loaded drug. ${ }^{42}$ Two molar percentages of PEGDA crosslinker were utilised ( 1 and $3 \%$ ) to observe the effects of crosslinking density ${ }^{43}$ during hydrogel characterisation.

Initial studies considered pNIPAAM for polymerisation instead of NIPAAM monomers. The aim was to polymerise HA-Tyr in the presence of pNIPAAM to produce a semi-IPN hydrogel. The gels lacked stability (liquid-like consistency) though the method did form hydrogels. Hydration of the gels resulted in leaching out of the pNIPAAM into the hydration media resulting in a cloudy solution when placed at $37^{\circ} \mathrm{C}$ (T>LCST). Due to the large size of pNIPAAM and HA-Tyr better mixing was thought to resolve this issue. A frost-defrost method ${ }^{44}$ was used to improve mixing as the defrost process ensues better contact of two large molecules. However, this method was found not to improve the stability issue of the pNIPAAM gels, therefore,, the results discussed in this study are based on hydrogels fabricated from NIPAAM monomers

\section{Characterisation of hydrogels}

\section{IR spectroscopy and scanning electron microcopy (SEM)}

FTIR results of HA-Tyr and PEGDA-pNIPAAM-HA hydrogels, NIPAAM monomer and $\mathrm{HA}$ are shown in Figure 1. Formation of PEGDA-pNIPAAM-HA gels is indicated by the disappearance of peaks which are characteristic of NIPAAM monomer, including $\mathrm{C}=\mathrm{C}$ $\left(1620 \mathrm{~cm}^{-1}\right)$ and $-\mathrm{CH}=\mathrm{CH} 2\left(960\right.$ and $\left.985 \mathrm{~cm}^{-1}\right)$. The broad C-O stretch attributable to HA occurs between $1050-1150 \mathrm{~cm}^{-1}$. Both HA and NIPAAM have a $\mathrm{C}=\mathrm{O}$ stretch that usually occurs between $1600-1620 \mathrm{~cm}^{-1}$. However, the appearance of a sharp peak at $1550 \mathrm{~cm}^{-1}$ in PEGDA-pNIPAAm-HA gel (absent in HA only) suggests an N-H stretch associated with the amide bond in NIPAAM. ${ }^{28}$ The sharp peak seen in NIPAAM at $3400 \mathrm{~cm}^{-1}$ represents $\mathrm{N}-\mathrm{H}$ in NIPAAM, which broadens in the gel suggesting possible interaction with the broad peak attributed to $\mathrm{O}-\mathrm{H}$ vibration in $\mathrm{HA}$. The spectrum for HATyr hydrogel remained largely similar to the spectrum of HA only. However, there is a visible peak at $2950 \mathrm{~cm}^{-1}$, which represents an aromatic $\mathrm{C}-\mathrm{H}$ stretch introduced by the Tyr group in HA-Tyr.

Microscopic investigation of PEGDA-pNIPAAM-HA gels showed a structure with multiple recurring, well-defined pores of around 1-2 $\mu \mathrm{m}$ (Figure 2A). HA-Tyr hydrogels possessed areas of smoother surface with some areas of rough surface and irregular, limited larger pores (Figure 2B). Porosity may be attributed to the freezedrying process. The loss of initially formed ice crystals creates multiple openings within the gel structure. Investigation into the effect of crosslinking density was not conducted, however, higher crosslink density was expected to result in less porous gels. ${ }^{21}$

Figures 1 and 2 


\section{Lower critical solution temperature (LCST)}

The thermoresponsiveness of pNIPAAM based gels at physiological temperature is driven by collapse to form a more hydrophobic gel at a glass transition midpoint $(\mathrm{Tg})$ known as the LCST. ${ }^{45}$ DSC was used to determine the LCST of the prepared pNIPAAM hydrogels with and without INF.

The LCST was $31.4 \pm 0.2$ (heat flow: $0.020 \mathrm{~W} / \mathrm{g}$ ) and $34.2 \pm 0.1^{\circ} \mathrm{C}$ (heat flow: $0.008 \mathrm{~W} / \mathrm{g}$ ) for 1 and $3 \%$ of pNIPAAM-PEGDA-HA hydrogels respectively (Figure $3 \mathrm{~A}$ ). In a previous study conducted in our laboratory, LCST values ranged from $34.3 \pm 0.2$ to $36.2 \pm 0.2^{\circ} \mathrm{C}(\mathrm{p}<0.05)$ for PEGDA amounts from $2-6 \%$ (with pNIPAAM-PEGDA hydrogels). ${ }^{32}$ Isopropyl groups predominate above the LCST leading to an aggregation mechanism that results in the overall domination of hydrophobicity. ${ }^{46}$ The polymer chain retracts from the surrounding solvent in a repulsive force manner. There is an expulsion of water content followed by a slower release of contents above the LCST.47

LCST values of $34.1 \pm 0.1$ and $35.7 \pm 0.3^{\circ} \mathrm{C}$ were recorded for 1 and $3 \%$ pNIPAAM-PEGDA-HA-INF hydrogels (Figure 3B). Protein loading resulted in broader response peaks/Tg. The slight increase in the LCST is thought to be due to the hydrophilic nature of INF. ${ }^{14,29,48,49}$ Interestingly the less crosslinked gels (both with and without protein) appeared to show sharper thermoresponsiveness seen as higher change in heat flow $(0.0070 \mathrm{~W} / \mathrm{g}$ cf. $0.0037 \mathrm{~W} / \mathrm{g})$. pNIPAAM-PEGDA-HA gels appeared clear at room temperature $\left(25^{\circ} \mathrm{C}\right)$ and visibly changed to a white gel when placed above its LCST (Figure 3C).

\section{Figure 3}

\section{Rheological behavior of hydrogels}

Rheological properties were expressed as two dynamic mechanical properties in this study i.e. elastic and viscous modulus. Elastic modulus $\left(G^{\prime}\right)$ refers to the energy stored within a gel that has been exposed to deformation i.e. oscillatory shear in this case. It is a measure of the elasticity of gels or the rigidity of gels derived from elastic deformation. Viscous modulus $\left(G^{\prime \prime}\right)$ refers to the energy lost in a material following deformation. ${ }^{50}$

$G^{\prime}$ was found to be higher than $G^{\prime \prime}$ in all the empty gels produced suggesting that the $G^{\prime}$ pre-dominates and therefore, all gels demonstrated properties of a viscoelastic solid ${ }^{51}$ (Figures $4 \mathrm{~A}$ and $\mathrm{B}$ ). Higher cross-linking concentrations (5\% HATyr and 3\% PEGDA-pNIPAAM-HA gels) contributed to higher moduli and therefore, higher overall rigidity. Increased frequency of oscillation had little influence on the moduli of highly crosslinked hydrogels (5 \% HA-Tyr and $3 \%$ PEGDA-pNIPAAM-HA). 
Although all the prepared gels were injectable, it is expected that the injectablility of gels inversely correlates with the overall rigidity of the gels $\left(G^{\star}\right){ }^{52}$

A significant drop in $G^{\prime \prime}$ was observed for all the INF loaded hydrogels, especially with $1 \%$ PEGDA-pNIPAAM-HA-INF and 1\% HA-Tyr-INF. These two hydrogels also showed a higher frequency dependent change in moduli. Interestingly, 1\% PEGDA-pNIPAAM-HA-INF showed a drop in G" (Figure 4B) at the highest oscillation frequency suggesting a higher tendency to "yield" when such a large molecule such as INF is incorporated within the matrix (perhaps due to increased disentanglement within the gel network).

Figure $4 \mathrm{C}$ shows the temperature-dependent change in the moduli $\left(G^{*}\right)$ of pNIPAAM-based hydrogels. A steep increase in $G^{*}$ is seen at approximately $33^{\circ} \mathrm{C}$ with the empty gels and $34^{\circ} \mathrm{C}$ with the INF-loaded hydrogels. The increased moduli is attributed to the temperature-dependent sol-gel transition of pNIPAAM when T>LCST causing its gels to have higher $G^{*}$ compared to HA-Tyr gels, which are not thermosensitive. Overall, this rheology study showed that higher crosslink density led to increased rigidity of the hydrogels and that the incorporation of INF within the gels only had an influence in the moduli of lower cross-linked hydrogel formulation, which demonstrated shear thinning behaviour.

\section{Figure 4}

\section{Swelling ratio (SR) measurements}

SR gives potential insight into the release kinetics of drugs loaded within hydrogels and mechanical structure of the hydrogels. ${ }^{21}$ Empty HA-Tyr hydrogels showed higher SR values compared to pNIPAAM hydrogels at both 25 and $37^{\circ} \mathrm{C}$ (Figure $5 \mathrm{~A}$ ). The higher SR of HA-Tyr can be due to its multiple $\mathrm{OH}$ groups, ${ }^{12}$ which can lead to stronger attraction with water molecules as compared to the hydrophobic isopropyl groups present in NIPAAM.

A SR of $56.5 \pm 0.1$ and $49.6 \pm 0.8$ was observed for the 1 and $5 \% \mathrm{HA}-\mathrm{Tyr}$ hydrogels respectively, whereas a SR of $24.2 \pm 1.2$ and $20.5 \pm 1.4$ was seen for 1 and $3 \%$ pNIPAAM-HA hydrogels at $25^{\circ} \mathrm{C}$ respectively. There was no significant difference $(p>0.05)$ in the intra SR i.e. within the pNIPAAM-HA or HA-Tyr hydrogels. However, there was a significant difference $(p<0.05)$ in swelling when gels are inter-compared.

A SR of $4.2 \pm 1.2$ and $3.7 \pm 1.8$ for empty 1 and $3 \%$ pNIPAAM-HA hydrogels was observed at $37^{\circ} \mathrm{C}$ respectively. There were significant differences in $\operatorname{SR}(p<0.05)$ when the temperature increased to $37^{\circ} \mathrm{C}$. The drop in SR can be attributed to the collapsing property of pNIPAAM. A rearrangement occurs at $37^{\circ} \mathrm{C}$ causing the hydrophobic isopropyl group to predominate leading to an expulsion of water content 
previously contained in the hydrogel. ${ }^{45}$ An average SR of $53.2 \pm 2.6$ and $51.7 \pm 6.8$ was observed for 1 and 5\% HA-Tyr gels respectively indicating that there was no significant difference $(p<0.05)$ in the $S R$ at $37^{\circ} \mathrm{C}$.

The incorporation of INF $(5.0 \mathrm{mg} / \mathrm{mL})$ reduced the $S R$ in all hydrogel formulations (Figure 5B). The reduction in residential space for water in presence of INF within the hydrogel pores could have contributed to the decrease in SR. ${ }^{4}$ Another possible reason could be due to INF mediated disruption of interaction with water molecules. A SR of $29.4 \pm 0.3: 31.8 \pm 1.9$ was seen with $1 \% \mathrm{HA}-\mathrm{Tyr}$ hydrogel at $25: 37^{\circ} \mathrm{C}$ respectively, $10.2 \pm 0.1: 10.0 \pm 0.2$ for $5 \%$ HA-Tyr-INF hydrogels respectively. There was no significant difference $(p>0.05)$ in $S R$ of each hydrogel at these two temperatures. A SR of $21.5 \pm 0.7: 1.7 \pm 0.2$ was seen with $1 \%$ pNIPAAM-HA-INF at $25: 37^{\circ} \mathrm{C}$ respectively, whereas a SR of $8.3 \pm 0.2: 2.1 \pm 0.1$ for $3 \%$ PNIPAAM-HA-INF respectively. The reduction in SR was significant $(p<0.05)$, which could be attributed to the thermoresponsive properties of pNIPAAM.

Figure 5

\section{In vitro release kinetics using the PK-Eye}

Experiments using the PK-Eye ${ }^{40}$ were conducted to determine which gel formulation could effectively extended the release profile of INF. The PK-Eye is a two-compartment model that mimics certain properties of the human eye. ${ }^{40}$ One key advantage of the PK-Eye was that it provides a distinct outflow so that the quantification of INF could be easily calculated. The pump flow system provided fresh buffer as opposed to a singlecompartment (non-outflow) release system that involved dilution of the drug with each sampling due to addition of fresh buffer for volume correction. INF (1.0 mg, $200 \mu \mathrm{L})$ loaded hydrogels were injected in the posterior cavity of the PK-Eye containing PBS $(\mathrm{pH} 7.4)$ and outflow samples from the anterior cavity was analysed with the BCA assay.

An initial screening study was conducted to observe the difference in INF release kinetics. INF released was $74.2 \pm 0.4$ and $71.4 \pm 0.2 \%$ for the 1 and $5 \% \mathrm{HA}$ Tyr gels respectively by day 12 (top panel, Figure 6 ). INF released was $50.4 \pm 0.8$ and $40.9 \pm 0.5 \%$ for the 1 and $3 \%$ pNIPAAM-HA gels respectively by day 12 (no significant difference). The data generated also suggests that crosslinking had not exceeded a point where the hydrogels possess such a dense structure that loading of INF becomes significantly lower.

Following the screening study, two formulations were selected for repeated release studies (bottom panel, Figure 6). Selection was conducted based on the 
rheology of the formulations since cross-linking density did not appear to significantly impede the release kinetics of each type of hydrogel. Gels with lower cross linking were preferred for the release study on the basis of overall lower moduli (Figure 4) being related to improved injectability. However, the 1\% HA-Tyr-INF hydrogel was discontinued for further release studies due to its almost full liquid-like state, which affected handling (e.g. washing step and further characterisation). Confirmed removal of cross-linking reagents will be necessary for further development.

The release study (bottom panel, Figure 6) demonstrated a similar 2:1 (approximate) release trend seen in the initial screening (top panel, Figure 6). INF released was $45.4 \pm 0.3$ and $24.9 \pm 0.4 \%$ at day 9 for $5 \%$ HA-Tyr and $1 \%$ PEGDAHA-pNIPAAM respectively. The difference in percentage protein release was statistically significant $(p=0.0125, n=3)$. The anterior concentration of INF (Figure 6) suggests that $1 \%$ PEGDA-pNIPAAM-HA-INF showed less of a burst release, with concentration values of $10-20 \mu \mathrm{g} / \mathrm{mL}$ throughout the 9 days.

The results were promising as the experiment was conducted in PBS and it is expected that proteins will diffuse more slowly in a more viscous environment (such as simulated vitreous). ${ }^{40}$ Previous studies of ranibizumab ( $50 \mathrm{kDa}$ ) and bevacizumab (150 $\mathrm{kDa}$ ) in PBS have a reported $t_{1 / 2}$ of $1.5 \pm 0.6$ and $1.2 \pm 0.1$ days respectively with almost 90-100\% protein clearance within 7-10 days. ${ }^{40}$ These results indicate that INF (150 $\mathrm{kDa}$ ) loaded hydrogels do show sustained release in the posterior cavity. The difference in SR of both HA-Tyr and PEGDA-pNIPAAM-HA hydrogels could have contributed to the faster INF release in the former. HA-Tyr gels had a higher SR (Figure 5B) than PEGDA-pNIPAAM-HA based gels. Swelling may be seen as a dissolution mechanism that promotes the osmotic drive of encapsulated INF from the hydrogel into the release media. ${ }^{21}$ The physiochemical property of pNIPAAM is such that above LCST, it shrinks due to domination of hydrophobic groups. Essentially the collapse of pNIPAAM gels appears to result in a depot-like effect resulting in an even slower release of INF ${ }^{4}$ perhaps due to the erosion of the gel. The slow release of pNIPAAM hydrogel is well known and it has been proposed that grafting NIPAAM with a hydrophilic compound would be necessary to hasten the release of drug loaded. ${ }^{53}$

\section{Figure 6}

\section{Conclusions}

One of the most important goals in ophthalmic drug development is to extend the therapeutic level of drugs, especially proteins and antibodies, in the posterior segment to treat blinding diseases. With an ageing population and with people living longer, there is a need to develop extended formulations for ocular use. In situ-forming 
injectable gels have long been examined to encapsulate drugs for sustained local release. Hydrogels when used in a drug delivery system to prolong the release of a drug typically contain a lot of water and are often burdened by an initial burst release of the drug. The release of a drug in a burst can be of an advantage clinically as a 'loading dose', but is more often a major concern because it may shorten total duration of release and result in dose-dependent adverse effects. HA-Tyr and PEGDApNIPAAM-HA were prepared and loaded with INF as a model protein. Thermoresponsive properties of pNIPAAM-based hydrogels were retained even after the addition of INF. The crosslinking density of the hydrogels correlated with SR and overall modulus of the gels. Highly crosslinked gels had a lower SR but showed more rigidity. The PK-Eye helps estimate the release profiles of long lasting dosage forms during preclinical research that may accelerate development. The PK-Eye was designed to be simple and practical to use to allow for iterative processes to occur so that formulations can be optimised efficiently and while minimising the use of animal models for suboptimal preclinical candidates. All hydrogels showed sustained release profiles as compared to the protein alone (previously tested) when conducted in the PK-Eye. PEGDA-pNIPAAM-HA showed slower release profile as compared to HA-Tyr hydrogels.

\section{Acknowledgements}

We are grateful for funding from the National Institute of Health Research (NIHR) Biomedical Research Centre at Moorfields Eye Hospital NHS Foundation Trust and UCL Institute of Ophthalmology, Moorfields Special Trustees, the Helen Hamlyn Trust (in memory of Paul Hamlyn), Medical Research Council, Fight for Sight and the Michael and Ilse Katz foundation. R.E. is grateful for funding from the EPSRC in the form of a studentship under the EPSRC Centre for Doctoral Training in Targeted Therapeutics and Nanomedicines (EP/L01646X/1). The research was also undertaken at UCL/UCLH which receives funding from the Department of Health's NIHR as a Comprehensive Biomedical Research Centre.

\section{References}

1. del Amo EM, Urtti A. Current and future ophthalmic drug delivery systems. A shift to the posterior segment. Drug Discov Today. 2008;13(3-4):135-143.

2. Rauck BM, Friberg TR, Medina Mendez C a., et al. Biocompatible reverse thermal gel sustains the release of intravitreal bevacizumab in vivo. Investig Ophthalmol Vis Sci. 2013;55(1):469-470.

3. Vaishya R, Khurana V, Patel S, Mitra AK. Long-term delivery of protein 
therapeutics. Expert Opin Drug Deliv. 2015;12(3):415-440.

4. Hoare TR, Kohane DS. Hydrogels in drug delivery: Progress and challenges. Polymer (Guildf). 2008;49(8):1993-2007.

5. Dueramae I, Nishida M, Nakaji-Hirabayashi T, Matsumura K, Kitano H. Biodegradable shape memory polymers functionalized with anti-biofouling interpenetrating polymer networks. J Mater Chem B. 2016;4(32):5394-5404.

6. Dragan ES. Design and applications of interpenetrating polymer network hydrogels. A review. Chem Eng J. 2014;243:572-590.

7. Shu XZ, Liu Y, Palumbo FS, Luo Y, Prestwich GD. In situ crosslinkable hyaluronan hydrogels for tissue engineering. Biomaterials. 2004;25(7-8):13391348.

8. Ha DI, Lee SB, Chong MS, Lee YM, Kim SY, Park YH. Preparation of thermoresponsive and injectable hydrogels based on hyaluronic acid and poly $(\mathrm{N}$ isopropylacrylamide) and their drug release behaviors. Macromol Res. 2006;14(1):87-93.

9. Baino F. Towards an ideal biomaterial for vitreous replacement: Historical overview and future trends. Acta Biomater. 2011;7(3):921-935.

10. Saettone MF, Monti D, Torracca MT, Chetoni P. Mucoadhesive ophthalmic vehicles: evaluation of polymeric low-viscosity formulations. J Ocul Pharmacol. 1994;10(1):83-92.

11. Balazs EA, Denlinger JL. Clinical uses of hyaluronan. Ciba Found Symp. 1989;143:265-75-80, 281-285.

12. Pescosolido L, Schuurman W, Malda J, et al. Hyaluronic acid and dextranbased semi-IPN hydrogels as biomaterials for bioprinting. Biomacromolecules. 2011;12(5):1831-1838.

13. Cho KY, Chung TW, Kim BC, et al. Release of ciprofloxacin from poloxamergraft-hyaluronic acid hydrogels in vitro. Int J Pharm. 2003;260(1):83-91.

14. Stile RA, Burghardt WR, Healy KE. Synthesis and characterization of injectable poly ( $\mathrm{N}$-isopropylacrylamide)-based hydrogels that support tissue formation in vitro. Macromolecules. 1999;32(22):7370-7379.

15. Mayol L, Quaglia F, Borzacchiello A, Ambrosio L, Rotonda MI La. A novel poloxamers/hyaluronic acid in situ forming hydrogel for drug delivery: Rheological, mucoadhesive and in vitro release properties. Eur $J$ Pharm Biopharm. 2008;70(1):199-206.

16. Lian S, Xiao Y, Bian $Q$, et al. Injectable hydrogel as stem cell scaffolds from the thermosensitive terpolymer of NIPAAm/AAc/HEMAPCL. Int $J$ Nanomedicine. 2012;7:4893-4905. 
17. Lee F, Chung JE, Kurisawa M. An injectable hyaluronic acid-tyramine hydrogel system for protein delivery. J Control Release. 2009;134(3):186-193.

18. Chin L, Calabro A, Walker E, Derwin KA. Mechanical properties of tyramine substituted-hyaluronan enriched fascia extracellular matrix. J Biomed Mater Res - Part A. 2012;100 A(3):786-793.

19. Kontturi LS, Järvinen E, Muhonen V, et al. An injectable, in situ forming type II collagen/hyaluronic acid hydrogel vehicle for chondrocyte delivery in cartilage tissue engineering. Drug Deliv Trans/ Res. 2014;4(2):149-158.

20. Yu Y, Lau LCM, Lo AC-Y, Chau Y. Injectable Chemically Crosslinked Hydrogel for the Controlled Release of Bevacizumab in Vitreous: A 6-Month In Vivo Study. Trans/ Vis Sci Technol. 2015;4(2):5.

21. Bae KH, Wang L-S, Kurisawa M. Injectable biodegradable hydrogels: Progress and challenges. J Mater Chem B. 2013;1(40):5371-5388..

22. Kurisawa M, Chung JE, Yang YY, et al. Injectable biodegradable hydrogels composed of hyaluronic acid tyramine conjugates for drug delivery and tissue engineering. Chem Commun. 2005;53(34):4312.

23. Darr A, Calabro A. Synthesis and characterization of tyramine-based hyaluronan hydrogels. J Mater Sci Mater Med. 2009;20(1):33-44.

24. Hilborn J. In vivo injectable gels for tissue repair. Wiley Interdiscip Rev Nanomedicine Nanobiotechnology. 2011;3(6):589-606.

25. Loebel C, Broguiere N, Alini M, Zenobi-Wong M, Eglin D. Microfabrication of photo-cross-linked hyaluronan hydrogels by single- and two-photon tyramine oxidation. Biomacromolecules. 2015;16(9):2624-2630.

26. Turturro SB, Guthrie MJ, Appel AA, et al. The effects of cross-linked thermoresponsive PNIPAAm-based hydrogel injection on retinal function. Biomaterials. 2011;32(14):3620-3626.

27. Xu X-D, Wei H, Zhang X-Z, Cheng S-X, Zhuo R-X. Fabrication and characterization of a novel composite PNIPAAm hydrogel for controlled drug release. J Biomed Mater Res A. 2007;81(2):418-426.

28. Zhao Z, Li Z, Xia Q, Xi H, Lin Y. Fast synthesis of temperature-sensitive PNIPAAm hydrogels by microwave irradiation. Eur Polym J. 2008;44(4):12171224.

29. Wu JY, Liu SQ, Heng PWS, Yang YY. Evaluating proteins release from, and their interactions with, thermosensitive poly ( $\mathrm{N}$-isopropylacrylamide) hydrogels. J Control Release. 2005;102(2):361-372.

30. Kang Derwent JJ, Mieler WF. Thermoresponsive hydrogels as a new ocular drug delivery platform to the posterior segment of the eye. Trans Am Ophthalmol 
Soc. 2008;106:206-213-214.

31. Drapala PW, Brey EM, Mieler WF, Venerus DC, Kang Derwent JJ, Pérez-Luna VH. Role of Thermo-responsiveness and Poly(ethylene glycol) Diacrylate Cross-link Density on Protein Release from Poly(N-isopropylacrylamide) Hydrogels. J Biomater Sci Polym Ed. 2011;22(1-3):59-75.

32. Awwad S, Al-Shohani A, Khaw PT, Brocchini S. Comparative Study of In Situ Loaded Antibody and PEG-Fab NIPAAM Gels. Macromol Biosci. 2017:1700255.

33. Bertrand N, Fleischer JG, Wasan KM, Leroux JC. Pharmacokinetics and biodistribution of $\mathrm{N}$-isopropylacrylamide copolymers for the design of $\mathrm{pH}$ sensitive liposomes. Biomaterials. 2009;30(13):2598-2605.

34. Yu L, Ding J. Injectable hydrogels as unique biomedical materials. Chem Soc Rev. 2008;37(8):1473-1481.

35. Wu Y, Yao J, Zhou J, Dahmani FZ. Enhanced and sustained topical ocular delivery of cyclosporine $A$ in thermosensitive hyaluronic acid-based in situ forming microgels. Int J Nanomedicine. 2013;8:3587.

36. Widjaja LK, Bora M, Chan PNPH, Lipik V, Wong TTL, Venkatraman SS. Hyaluronic acid-based nanocomposite hydrogels for ocular drug delivery applications. J Biomed Mater Res - Part A. 2014;102(9):3056-3065.

37. Cordero-Coma M, Yilmaz T, Onal S. Systematic Review of Anti-Tumor Necrosis Factor-alpha Therapy for Treatment of Immune-mediated Uveitis. Ocul Immunol Inflamm. 2013;21(1):19-27.

38. Posarelli C, Arapi I, Figus M, Neri P. Biologic agents in inflammatory eye disease. J Ophthalmic Vis Res. 2011;6(4):309-316.

39. Mirshahi A, Hoehn R, Lorenz K, Kramann C, Baatz H. Anti-tumor necrosis factor alpha for retinal diseases: current knowledge and future concepts. $J$ Ophthalmic Vis Res. 2012;7(1):39-44.

40. Awwad S, Lockwood A, Brocchini S, Khaw PT. The PK-Eye: A Novel In Vitro Ocular Flow Model for Use in Preclinical Drug Development. J Pharm Sci. 2015;104(10):3330-3342.

41. Ueda K, Akiba J, Ogasawara S, et al. Growth inhibitory effect of an injectable hyaluronic acid-tyramine hydrogels incorporating human natural interferon-?? and sorafenib on renal cell carcinoma cells. Acta Biomater. 2016;29:103-111.

42. Liow SS, Dou Q, Kai D, et al. Thermogels: In Situ Gelling Biomaterial. ACS Biomater Sci Eng. 2016;2(3):295-316.

43. Mwangi JW, Ofner CM. Crosslinked gelatin matrices: Release of a random coil macromolecular solute. Int J Pharm. 2004;278(2):319-327.

44. Jin S, Liu M, Zhang F, Chen S, Niu A. Synthesis and characterization of pH- 
sensitivity semi-IPN hydrogel based on hydrogen bond between poly $(\mathrm{N}$ vinylpyrrolidone) and poly(acrylic acid). Polymer (Guildf). 2006;47(5):1526-1532.

45. Klouda L. Thermoresponsive hydrogels in biomedical applications A seven-year update. Eur J Pharm Biopharm. 2015;97:338-349.

46. Cao Z, Liu W, Gao P, Yao K, Li H, Wang G. Toward an understanding of thermoresponsive transition behavior of hydrophobically modified $\mathrm{N}$ isopropylacrylamide copolymer solution. Polymer (Guildf). 2005;46(14):52685277.

47. Gandhi A, Paul A, Sen SO, Sen KK. Studies on thermoresponsive polymers: Phase behaviour, drug delivery and biomedical applications. Asian J Pharm Sci. 2015;10(2):99-107.

48. Overstreet DJ, Dhruv HD, Vernon BL. Bioresponsive Copolymers of Poly (Nisopropylacrylamide) with Enzyme-Dependent Lower Critical Solution Temperatures. Biomacromolecules. 2010;11:1154-1159.

49. Wu XY, Lee PI. Preparation and Characterization of Thermal- and $\mathrm{pH}$-Sensitive Nanospheres. Pharm Res. 1993;10(10):1544-1547.

50. Seddiki N, Aliouche D. Synthesis, rheological behavior and swelling properties of copolymer hydrogels based on poly(n-isopropylacrylamide) with hydrophilic monomers. Bull Chem Soc Ethiop. 2013;27(3):447-457.

51. Kang EY, Moon HJ, Joo MK, Jeong B. Thermogelling chitosan-g-(PAF-PEG) aqueous solution as an injectable scaffold. Biomacromolecules. 2012;13(6):1750-1757.

52. Supper S, Anton N, Boisclair J, et al. Chitosan/glucose 1-phosphate as new stable in situ forming depot system for controlled drug delivery. Eur $J$ Pharm Biopharm. 2014;88(2):361-373.

53. Gil ES, Hudson SM. Effect of silk fibroin interpenetrating networks on swelling/deswelling kinetics and rheological properties of poly $(\mathrm{N}$ isopropylacrylamide) hydrogels. Biomacromolecules. 2007;8(1):258-264. 